\title{
PROBLEMAS \\ EPISTEMOLÓGICOS DE LAS CIENCIAS HUMANAS Y DE LA EDUCACIÓN
}

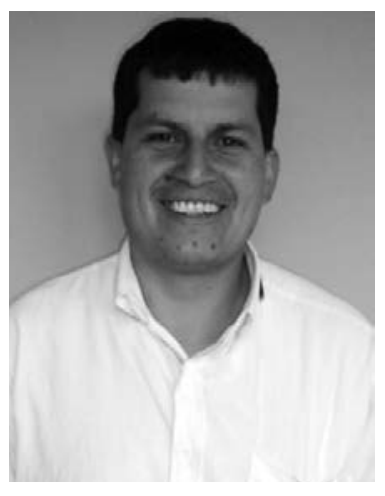

Darwin Reyes Solís*

Zl campo de la epistemología es un mundo de conflictos, ¿en ese sentido es mejor plantearse el problema epistemológico desde la visión de un sector de diferencias y luchas constantes, de un mundo de diversidad en el que las posiciones llegan a ser extremas, posiciones que van desde la negación de la epistemología como ideología científica, hasta afirmaciones de la epistemología como la posibilidad de un marco referencial único para el conocimiento humano validado como científico.

No existe una concepción homogénea y única sobre lo que es la epistemología y sobre su campo u objeto de estudio. Más bien la epistemología es un continuo debate sobre su propio corpus teórico. Podemos hablar en sentido estricto de epistemologías, de diversidad de concepciones. Esta diversidad dentro de la epistemología depende de preconcepciones sobre la ciencia, el conocimiento y los saberes; depende de las preconcepciones políticas y sociales sobre lo que implican, esos saberes, en sus aplicaciones y usos.

La concepción misma de la epistemología es un problema teórico y pragmático. Además, las diferentes formas de entenderse a sí misma han estado atadas al proceso histórico. Los cambios culturales van haciendo que los planteamientos epistemológicos vayan de la mano con las transformaciones sociales. Ya desde su origen la concepción de episteme fue creada para diferenciarse de otras formas de entender el mundo, para diferenciarse de la religión, de los mitos, de lo cotidiano, para así, en esa diferenciación, lograr un estatus de "verdad”, un estatus de "logos” que se construye desde un tipo de racionalidad, que se creía como única.

Pero es en la modernidad que las ciencias se imponen como "estructura”. Desde allí se puede hablar de epistemología en el sentido de teoría de las ciencias, teoría crítica y reflexiva sobre la construcción del conocimiento científico. Además la modernidad piensa la epistemología desde concepciones de teoría "para" las ciencias naturales. Podemos decir que la concepción moderna de la epistemología tiene su base en el debate de los presupuestos de las ciencias formales (matemática y lógica) y las ciencias naturales reinadas por la física. Teniendo claro que esa era la línea predominante en la modernidad, pero no la única.

Las superaciones a esas formas de entender la epistemología han venido de diferentes planteamientos, de diferentes formas de volver los ojos a las construcciones científicas. Como ejemplos ubiquemos a los planteamientos nietzcheanos y sus superaciones de lo "Apolíneo" desde el surgimiento de lo “dionisiaco” como categorías simbólicas del problema entre la racionalidad y las estructuras de diferencia; el planteamiento hermenéutico, las construcciones sobre conocimientos étnicos, la genealogía y su construcción de biopoder, los planteamientos de estudios subalternos y postcoloniales, la recostrucción y sus presupuestos, las

* Docente de la Facultad de Ciencias Humanas y de la Educación de la Universiad Politécnica Salesiana. 
teorías de la complejidad, la teoría de los sistemas sociales entre muchos otros.

La diversidad de planteamientos epistemológicos en la actualidad cubre un rango extenso. Las propuestas están dadas desde construcciones históricas más conservadoras en términos conceptuales, hasta el anarquismo epistemológico. Todo esto me lleva a afirmar que el primer "problema” epistemológico es la comprensión sobre sí misma, la comprensión de lo que se entiende por epistemología. Dependerá en mucho, de la concepción de epistemología que se esté manejando para definir sus conflictos internos, sus concepciones teóricas y sus implicaciones prácticas.

\subsection{El ser mismo de la epistemología}

Uno de los campos de conflicto de la epistemología está dado en las tendencias contemporáneas de "alivianamiento" de los presupuestos teóricos. Llevando la epistemología a un campo se subjetividades y planteamientos relativos logrados desde una anulación de la fundamentación teórica y una anulación de los presupuestos de la crítica y la coherencia exigida por cualquier construcción cognitiva. Llegando a casos extremos de plantear que todo lo que se produce desde lo cotidiano es ciencia y la única forma de validación es la aceptación del grupo cultural. Este alivianamiento tiene su expresión extrema en el relativismo cultural que anula toda posibilidad de construcción formal de la realidad social, lleva a pensar que todo vale, y todo "conocimiento" tiene verdad en sí mismo, porque la subjetividad de un expositor es suficiente para acreditar la no falsedad de una verdad enunciada.

Pero al mismo tiempo que la relativización del conocer y el alivianamiento teórico son serios problemas, también es preocupación de la epistemología con- temporánea las absolutizaciones de la verdad científica. Esa percepción de que el modelo de las ciencias formales como la matemática y la lógica son los únicos marcos referenciales para hacer ciencia y el lenguaje formal matematizado es el único que permite "exponer" con certeza la realidad. Fuera de eso, todo es metafísica (en el sentido despectivo del uso de este término) Otra versión de este absolutismo es determinar que el conocimiento científico es el único válido, que tienen su propio método y que su validación está dada en sí mismo, por el nivel de "precisión” de su comprobación. Dicho único método es el científico que expresa cánones estructurales que los científicos deben seguir.

Los dos extremos al plantearse el problema epistemológico olvidan que la ciencia implica apelar a teorías, dichas teorías son preconcepciones desarrolladas críticamente y con establecimientos de coherencia interna, con contrastación empírica, con la explicitación de una o unas lógicas internas y con la posibilidad de ser comunicable. Todo eso hace necesario una rigurosidad metódica. Esto hace que las construcciones teóricas sean realizadas y realizables dentro de ciertas estructuras que no son subjetivas en su totalidad, pero que tampoco son verdades preconcebidas y absolutas, independientes del sujeto que las formula. En el fondo este es uno de los grandes problemas y retos que tienen que enfrentar la epistemología contemporanea: encontrar canales de conciliación entre lo particular, lo subjetivo, lo local y la estructura normativa general (aunque no absoluta) que permita la existencia de presupuestos; encontrar sistemas de diálogo entre lo que es la subjetividad, la relatividad de la proposiciones científicas y su validación coherente y crítica (válida) de una realidad social que puede ser "leída", una normatividad que permita entender las estructuras generales. 
En este punto podemos citar la propuesta de Morín:

La racionalidad debe reconocer la porción del afecto, del amor y del arrepentimiento. La verdadera racionalidad conoce los límites de la lógica, del determinismo, del mecanicismo; sabe que la mente humana no puede ser omnisciente, que la realidad comporta misterio; ella negocia con lo no racionalizado, lo oscuro, lo que no se puede racionalizar; no solo es crítica sino autocrítica. Reconocemos la verdadera racionalidad por su capacidad para reconocer sus insuficiencias.

La racionalidad no es una cualidad de la que están dotadas las mentes de los científicos y técnicos y de la cual carecen los demás. Los sabios atomistas, racionales en su área de competencia y bajo el apremio del laboratorio, pueden ser completamente irracionales en política o en su vida privada ${ }^{1}$.

En esto, el problema epistemológico, es parecido al problema ético: la conciliación del individuo con el grupo, el derecho de la persona y sus relaciones con el de la colectividad. Mis derechos con los derechos de los demás. La ética debe buscar conciliaciones entre estos dos ámbitos.

\subsection{Las divisiones de la epistemología}

En cuanto a la división de la epistemología se han ensayado varias posibilidades: la de ciencias fácticas y ciencias formales; la de ciencias naturales y ciencias sociales, entre muchas otras formas de clasificar. Pero cualquiera sea la clasificación es un problema que se debe debatir dentro del corpus teórico de la epistemología

\footnotetext{
1 MORÍN, Edgar. "Los siete saberes necesarios para la educación del futuro”. Barcelona, Paidós, 1999, p. 30-31.
}

misma. La clasificación que se acepte es ya un debate planteado y si no se acepta ninguna clasificación es también un debate que debe ser atendido desde la epistemología y no fuera de ella.

Nos interesa en este artículo preguntarnos no solo por la clasificación en ciencias naturales y sociales, sino por el fondo de la concepción que se maneja en esta concepción: la existencia de las ciencias sociales. Es fundamental trabajar epistemológicamente las interrogantes sobre las ciencias sociales mismas: ¿Se puede llamar a las ciencias sociales ciencias? ¿Cuáles son las diferencias entre las construcciones de las ciencias llamadas naturales y las llamadas sociales? ¿Hay un método específico para la construcción de las ciencias sociales o el método de las ciencias naturales puede ser usado para hacer ciencias sociales. Al igual que nos planteamos el perfil de la construcción epistemológica de las ciencias sociales, cada una de las construcciones que se erigen como ciencias deben desmontar sus propios sistemas teóricos e ir al fondo de sus presupuestos. En ese sentido la pedagogía que se piensa a sí misma como construcción científica debe buscar su sistema de presupuestos que le permita "re-crearse" como una de las ciencias sociales.

La problemática es grande y las formas y líneas teóricas para enfrentar dicha problemática es diversa. Además se añade el hecho de ver lo previo de las ciencias sociales y las implicaciones en lo político, en lo social; sus implicaciones en el proceso de conocimiento, del aprendizaje de ese conocimiento y de las posibilidades reales de enseñar los bagajes teóricos construidos; de su incidencia en el uso y manejo del poder desde unos centros en los cuales se produce ciencia hacia otros lugares-países que toman esas ciencias y nada más. 
Pero el debate contemporáneo va más allá. Se ubica en la crítica y/o aceptación de esta forma de entender la construcción de conocimiento desde parcelas de la realidad. El desarrollo de las clasificaciones se ha visto limitada por los apremiantes argumentos de teorías (por ejemplo la complejidad) de buscar conexiones e interconexiones entre las diferentes formas del saber humano. No se trata solo de encontrar puntos de diálogo entre las ciencias llamadas naturales y las ciencias sociales. Se trata de encontrar nodos de conexión entre los diferentes conocimientos y saberes y dentro de ellos como uno más el conocimiento científico. Relaciones que son necesarias para entender los procesos de sociedades que ya no tienen un centro de referencia, sino que funcionan desde multiplicidad de referencialidades. Por ejemplo es apremiante ver la necesidad de incluir los saberes religiosos, los saberes culturales, los saberes pensados desde las diferencias (de género, de edad, de etnia, etc.) y los saberes científicos en un diálogo en el que ninguno es fundante de los otros. En el que no existen conocimientos superiores, ni inferiores, sino solo diferentes.

\subsection{La necesidad de la inclusión de otras formas de saber (no científicos) y el diálogo de conocimientos}

Otro de los serios problemas que tiene la epistemología en sociedades de diferencias pronunciadas, de construcciones culturales diversas, de sistemas históricos de injusticia, es el debate sobre otros conocimientos que luchan por posicionarse en los marcos de validación social. Dichos conocimientos, llamados también saberes están construidos desde "otras lógicas", otras formas de entender el conocimiento mismo, son conocimientos históricos y, muchos de ellos, milenarios.

El uso mismo de los términos ciencia, conocimiento y saber han sido tan diver- sos que no podemos establecer un acuerdo sobre qué quiere decir, en sentido estricto, cada uno. Podemos decir que hemos caído en concepciones tan generales que dicen todo y nada a la vez, que pueden ser utilizados en sentidos tan amplios que todo puede entrar en ese concepto, perdiendo toda especificidad para el debate y la criticidad. Por ello la propuesta de definir ciencias, conocimientos y saberes se convierte en una tarea "al imposible". Lo que podemos es trabajar estas concepciones desde otras formas de construirlas, desde otros presupuestos para entenderlos, hacer un metacognición de ellas como concepciones. En esta metacognición, como campo previo a la comprensión de todos estos términos, es fundamental entender que son términos que vienen de diferentes tradiciones, que vienen de diferentes propuestas culturales y teóricas, que no pueden ser clasificables en presupuestos fuera de sus propuestas, porque perderían sus dimensiones en las cuales tenían sentidos construidos.

La alternativa, entonces, es no jerarquizarlos, no colocar una tradición sobre otra. Pues ese, parece, el complejo cientista o cultural que menos aporta para las construcciones de epistemes desde la diversidad; pues cuando se los jerarquiza, uno gana y otro pierde, dejando en evidencias prejuicios epistémicos y culturales a favor o en contra de una posición. En otras palabras la jerarquización de estos términos implica una preferencia por uno de ellos y eso evidencia la poca apertura a que conocimientos, saberes y ciencia sean construcciones del saber, del conocimiento y de la ciencia que aportan al entendimiento de lo complejo de la realidad, de lo diverso de las sociedades y de los mundos (en el sentido heidegeriano). No con esto queremos decir que todo vale, sino que los caminos son diversos, por ello hemos de ser críticos con las construcciones sobre "el mundo" que 
se hagan desde la ciencia, desde los saberes y desde los conocimientos.

Los debates sobre si es ciencia, si es conocimiento o si es saber quedan superadas desde el planteamiento de "diversidad epistémica”, pues esta, entendida como la multiplicidad de formas de construir y distribuir los productos epistémicos sobre el entendimiento del mundo, de la vida, de la sociedad, de nosotros y de los otros, se inscribe en un manantial de formas, en un arco iris de posibilidades hacia el infinito.

Sobre este punto dejamos pendiente alguna afirmación concluyente, pues es un debate que está siendo tratado por nuestras generaciones y las alternativas no están del todo trabajadas. Podemos plantearnos el tema del diálogo de conocimientos como una labor por construir.

Ahora bien, existen intelectuales que han preferido tomar partido y decir que esos conocimientos son científicos pero con otra lógica de construcción de la ciencia, al otro lado están los intelectuales que desconocen la posibilidad de ciencia en esos conocimientos y les dan un otro lugar para ser elaborados y analizados. En todo caso en los tiempos actuales esos conocimientos ya no son negados (por lo menos en las referencias teóricas). Las prácticas son otra historia.

\subsection{Las diferentes posiciones para "abrir" la epistemología hacia otros frentes}

Como vemos los problemas de la epistemología son centrales y atacan a su estructura interna misma. De allí que han surgido voces para abandonar la epistemología por considerarla una "lectura de occidente", por considerarla un campo cerrado frente a las necesidades actuales del mundo que se presenta como incierto y relativo.
Se plantea que es necesario superar las formas de entender la epistemología y confirmar otras construcciones sobre el saber y el conocer humano, para así trascender la modernidad y reinventar la realidad. Veamos algunos de los planteamientos en este sentido.

\subsubsection{La superación de la epistemolo- gía, para un trabajo en los epistemes que se manejan en la producción del conocimiento}

Las propuestas en este nivel buscan un punto de relación que permita abandonar la occidentalización de la ciencia y pasar a un encuentro de conocimientos desde sus producciones, desde sus puntos de surgimiento. Esos puntos de surgimiento no son científicos, sino culturales, sociales, de uso de poder, de intereses, de deseos presentes y ausentes.

La propuesta de Fernando Garcés, docente de epistemología e interculturalidad en la Universidad Politécnica Salesiana nos ayuda a entender mejor esta línea de pensamiento.

La concepción de epistemología forma parte de un cuerpo reflexivo que Occidente (sé que "occidente" es un término complicado pero está lo suficientemente generalizado como para dar una idea "amplia" que permita entender que se trata de aquello que históricamente se estructuró como Occidente de lo que era en aquél momento el centro de los sistemas interregionales más importantes previos al siglo $X V$, o sea, Europa central en referencia al mundo árabe-musulmán) ha ido refinando a lo largo de los últimos siglos. Es, por tanto, una concepción monocultural, geohistóricamente localizada $y$ referencialmente restringida. Es un concepto que tiene que ver con un tipo de conocimiento (particular) que en un momento de la historia rompió las fronteras de su ámbito de producción, imponién- 
dose como universal. Esto no quiere decir que no sea un concepto útil e importante pero debe ser visto, en mi opinión, desde este marco comprensivo y desde esta relatividad.

Marcado este espacio de comprensión de lo que debe ser entendido por epistemología, se la puede definir, en cualquiera de sus corrientes, con la afirmación general de que es la ciencia que se ocupa del estudio crítico del conocimiento científico o de la teoría del conocimiento. Lo cierto es que habría que pensar en las opciones de concepción desde dentro de este espacio provincial marcado por Occidente, o sea, lo que propiamente se llamaría epistemología, y otros espacios de producción del conocimiento o de epistemes (como un término de encuentro).

En el mundo existen diversos modos de producción del conocimiento y es necesario acercarse a esas epistemes para construir una epistemología que sea pluritópica, por lo menos. Pero al trabajar desde la idea misma de epistemología, ¿no significaría esto tratar de meter a las demás formas de producción del conocimiento en la lógica totalizadora y monopólica del saber de Occidente? Es posible. Sin embargo, dado que el conocimiento es siempre un conocimiento situado creo que se puede pensar en una especie de campo de intersección en el que se expresen en un cierto lenguaje mínimo esas distintas epistemes.

Desde los grupos subalternizados por Occidente creo que este es un ejercicio necesario dado el carácter político que tiene actualmente la necesidad de defender y reivindicar su conocimiento como propuesta alternativa de "humanidad" 2 .

2 Entrevista a Fernando Garcés, Universidad Politécnica Salesiana, grupo de epistemología de la carrera de filosofía, mayo del 2005. Versión no publicada.
Como vemos esta es una propuesta que busca integrar otros conocimientos y dialogar con la diversidad de producciones cognitivas, incluida la científica.

\subsubsection{La deconstrucción del cono- cimiento mismo}

En los tiempos actuales la epistemología está en la búsqueda de un "lugar de encuentro" desde el cual sea posible superar la reducción impuesta por la concepción de ciencia en el sentido racionalizante y racionalizador de la modernidad. Un paso hacia el fondo del problema, en el conocimiento mismo, en las estructuras del conocer. Se trata de un avance desde la decostrucción del conocer mismo, para descubrir sus errores en diferentes niveles y su capacidad de construirse como válido desde la racionalidad humana. Se reconoce, además que la producción de conocimientos es una producción cultural y social. La producción del conocimiento no es un problema de la ciencia, sino previo a ella, en la administración, en las políticas y en las estructuras mismas.

En efecto, el conocimiento no se puede considerar como una herramienta ready made que es posible utilizar sin examinar su naturaleza. El conocimiento del conocimiento debe aparecer como una necesidad primordial que serviría de preparación para hacer frente a riesgos permanentes de error y de ilusión que no cesan de parasitar la mente humana. Se trata de armar nuestras mentes para el combate vital hacia la lucidez.

Es necesario introducir y desarrollar en la educación el estudio de las características cerebrales, mentales y culturales del conocimiento humano, de sus procesos y modalidades, de las disposiciones tanto psíquicas como culturales que permiten arriesgar el error o la ilusión ${ }^{3}$.

3 Morín Edgar, op. Cit. Pág. 18-19. 
En la propuesta de Morín las construcciones cognitivas deben ser validadas desde el conocimiento mismo, en un ejercicio de autocrítica y reconocimiento de sus límites. Desde allí se trazan puentes de interrelación tanto entre las llamadas ciencias como entre los diferentes saberes que produce el mundo de lo humano.

\subsection{Las relaciones entre la producción de conocimiento y el poder}

La producción y distribución de conocimientos ha estado atada al manejo y uso de poder. Los grupos y sociedades manejan el conocimiento y la información como un proceso de acumulación. Una estructura pensada en la acumulación de conocimientos para la dominación del "otro". Estos ejercicios de matrimonio entre el conocimiento, entre la construcción de categorías teóricas y los grupos que toman las decisiones y poseen los bienes materiales de producción no es nueva. En Latinoamérica tenemos la experiencia de esto en la colonización de los españoles frente a las poblaciones nativas.

En este debate de la imposición de estructuras coloniales y cientistas en la formación de las ciencias y del manejo de poder desde esas ciencias Lander plantea un trabajo de "desvelamiento" de esa realidad para mostrar las estructuras que promulgan ese ocultamiento y proponer "salidas” desde los trabajos latinoamericanos. Para desvelar los sistemas eurocéntricos que naturalizaron la construcción liberal plantea dos dimensiones: 1) los estudios sobre las separaciones o particiones del mundo de lo real que hacían las estructuras europeas y 2) los estudios de las formas de articulación de los saberes modernos con la organización del poder. Luego de analizar estas dos dimensiones realiza propuestas o alternativas al pensamiento euro céntrico-colonial en América Latina: la construcción de un nuevo episteme desde América Latina basado en: la concepción de comunidad y participación, la liberación a través de la praxis, la relación sujeto-objeto desde la actoría social y la construcción del conocimiento, la pluralidad epistémica, la perspectiva de la resistencia ${ }^{4}$.

La pluralidad epistémica como forma de entender la realidad y el pensar sobre dicha realidad. La diferencia epistémica como categoría teórica y práctica que nos permite ver la realidad desde la multiplicidad de formas, desde el derroche de la vida en un manantial de construcciones desde lo distinto, desde lo que no es igual. Somos diferentes y construimos nuestros conocimientos, ciencias y saberes desde diferentes estructuras y formas. Hasta la realidad de nosotros mismos es imposible concebirnos como iguales, pues nuestros instantes históricos se construyen desde momentos diferentes, desde intereses diferentes y desde inconscientes de la diferencia.

Pensar las realidades desde la diversidad epistémica abre las posibilidades y se convierten en proyectos políticos, en luchas por el control de los símbolos que se constituyen en fundamentales y fundantes del avanzar de una sociedad.

Lander también plantea la relación entre crítica epistemológica y papel político y social de las ciencias, podemos decir que "es un error suponer que la crítica epistemológica al objetivismo y determinismo de la ciencia cartesiana, tiene que traducirse en un transformación o alteración significativa del papel político y cultural de la ciencia”.

4 Cfr. LANDER, Edgardo: “Ciencias sociales: saberes coloniales y euro céntricos”, en la colonialidad del saber: euro-centrismo y ciencias sociales. Perspectivas latinoamericanas, compilado por Edgardo Lander, Buenos Aires, CLACSO, 2000. 
Lander sostiene esta tesis criticando a Lyotard y da ejemplos históricos de esto: El caso de la física, de la relatividad y el principio de incertidumbre. "La experiencia en este siglo sugiere que el relativismo epistemológico no es incompatible con el ejercicio del poder a nombre de la verdad científica"5.

\subsection{La autoreferencialidad científica}

Para entrar en los debates epistemológicos consideramos necesario entender los debates sobre la modernidad. Pues es en la modernidad como espacio-tiempo que la epistemología se convierte en una disciplina que trabaja las concepciones de la ciencia y los conocimientos. Allí, desde Descartes se elabora el conocimiento como una relación entre un sujeto consciente que se pone al frente de una realidad inconsciente. Una concepción fijista y mecanicista de las formas de entender la realidad.

La importancia de estudiar la modernidad radica en que es en ese espacio de tiempo que se trabaja una concepción de ciencia y de producción de conocimientos que determinan la forma de un nuevo orden de las cosas a nivel mundial. Los fenómenos nuevos para el siglo XVI como el Renacimiento, la conquista de América y la conformación de estados hace que las formas de concebir la realidad y sus posibilidades de conocerlas creen una forma específica de conocer: la ciencia con su método que se erige como absoluto: el método científico.

La ciencia entonces se pone en el trono, desconoce a los otros manejos del conocer

5 LANDER, Edgardo: "Las trasformaciones postmodernas de la política”. Ponencia presentada en la XX Congreso de la asociación Latinoamericana de Sociología. México, 2 al 6 de octubre de 1995. Versión electrónica proporcionada por el autor. 1995. p. 7. y se ubica en una posición que le permite dominar sus propios sistemas de referencia. Una autoreferencialidad en la que es la misma ciencia que determina la validez o no validez de sus argumentos, de sus posibilidades de construcción. Esa autoreferencialidad hace que nada de fuera pueda intentar debatir con la ciencia, nada que no sea ciencia pueda dialogar con la ciencia. Así se intenta ser pura, anular los procesos históricos, los sistemas de poder, la intervención cultural, los inconscientes “activados” y otras interferencias al construir conocimiento.

El imaginario de una historia racional ascendente en la que solo se podía encontrar el bien, el futuro prometido como cielo en la tierra, permitía que la ciencia se presente como el camino para encontrar dicho cielo.

Pero, en las teorías contemporáneas estas formas de entender la ciencia y la historia se han derrumbado. A cambio la historia está vista como rupturas y la ciencia pura es una ilusión de engaño y poder. Si la historia es discontinua, entonces sus productos son discontinuos: la ciencia es discontinua, los mitos son discontinuos, mi vida y la de todos es discontinua, la felicidad, el miedo, el conciente es discontinuo. Para la epistemología es fundamental entender esto, tanto entender la modernidad como forma de entender la ciencia, como la destrucción de ese imaginario. Así podemos diferenciar superar dichos conflictos.

\section{CONCLUSIONES:}

- No existe una epistemología, sino una diversidad de construcciones epistemológicas, cuyos problemas fundamentales están dados en entender cuál es su corpus teórico, y cuáles sus límites.

- La epistemología se refiere a una o unas formas de conocer que posee la humani- 
dad, pero es imposible que pueda abarcar a todas. Pues ese "todas" es una gama infinita de formas de conocer y entender la realidad.

- La epistemología necesita de un continuo diálogo con otras formas de entender el conocimiento, debe saber de los límites de la razón y de los límites del conocer humano mismo. Debe abrir espacios de encuentro en los que "lo humano" funcione como espacio que nos permite construir y no solo destruir.

- Es importante rescatar que no hay recetas o definiciones sobre los temas planteados, eso sería reducir la riqueza del debate y definir una línea para transmitirla como verdadera. Los tópicos de la epistemología los debemos trabajar como debates, muchos de ellos sin solución, pero debates que son fundamentales para entender los procesos históricos sociales de la producción y distribución de las ciencias, los conocimientos y los saberes.

- El debate de la pedagogía debe insertarse en el debate del conocer humano en sus estructuras más fundamentales, tanto biológicas como psíquicas y culturales. Es mejor diseñar el aprendizaje y la enseñanza comprendiendo lo que está de fondo en las estructuras del conocer. 


\section{BIBLIOGRAFÍA.}

- Álvarez, Freddy, La crisis de la razón, Quito, Efímera, 1999.

- Dussel, Enrique, Hacia una filosofía política crítica, Bilbao, Desclée de Brouwer, 2001.

"Eurocentrismo y modernidad (Introducción a las lecturas de Frankfurt)", en capitalismo y geopolítica del conocimiento: el eurocentrismo y la filosofía de la liberación en el debate intelectual contemporáneo, compilado por Walter, Abya Yala, 2001.

- Fernández Marta y Barbosa Susana, Tendencias sociales y políticas contemporáneas, Buenos Aires, Hernandarias, 1996.

- Follari, Roberto, Epistemología y sociedad, Santa Fe, Homo Sapiens, 2000.

- Lander, Edgardo, "Las transformaciones postmodernas de la política”. Ponencia presentada en el XX Congreso de la Asociación Latinoamericana de Sociología. México, 2 al 6 de octubre de 1995. Versión electrónica. 1995

“Ciencias sociales: saberes coloniales y eurocéntricos”, en La colonialidad del saber: eurocentrismo y ciencias sociales. Perspectivas latinoamericanas, compilado por Edgardo Lander, Buenos Aires, CLACSO, 200

- Mardones J.M., Filosofía de las ciencias humanas y sociales, Barcelona, Anthropos, 1991.

- Martínez, Miguel, La investigación cualitativa etnográfica en educación, México, Trillas. 1998

- Morín, Edgar, Los siete saberes necesarios para la educación del futuro. Barcelona, Paidós, 1999.

Introducción al pensamiento complejo, Barcelona, Gedisa, 1990.

- Quijano, Aníbal, "Colonialidad del poder, eurocentrismo y América Latina”, en La colonialidad del saber: eurocentrismo y ciencias sociales. Perspectivas latinoamericanas, compilado por Edgardo Lander, Buenos Aires, CLACSO, 2000. 